\title{
MIRD: Radionuclide Data and Decay Schemes
}

K.F. Eckerman and A. Endo

Reston, VA: Society of Nuclear Medicine, 2008, 671 pages, $\$ 98$

This monograph, a collection of decay schemes of radionuclides relevant to nuclear medicine, includes 333 radionuclides of 87 elements and presents detailed abundance and energies of emissions from $\alpha$-particles, $\beta$-particles (negatrons and positrons), electron capture, internal transition, internal conversion, and $\gamma$-photons.

This edition is the second revision of the earlier MIRD scheme of 1989 updated with 91 new radionuclides and is supplemented with a resource compact disk containing continuous $\beta$-spectrums and diagrams for tailored extraction of data into analysis. The intended audience is scientists, physicians, and other practitioners of nuclear medicine. The emissions are truncated at below $0.01 \%$ of contributions to the total energies.

The second and third chapters provide an introductory explanation of radioactive decay and conventions as illustrated by selected examples. These chapters are followed by 630 pages of an exhaustive list of emission types, abundance, and energies grouped by elements. Most of the radionuclides of clinical interest are covered in this book. It is a handy and authoritative resource for researchers and is particularly useful for the design of radiopharmaceuticals for imaging, for the design of radionuclide therapies, and for radiation dosimetry calculations.

Franklin C. Wong

M.D. Anderson Cancer Center

1515 Holcombe Blvd., Unit 1264

Houston, TX 77030

E-mail: fwong@di.mdacc.tmc.edu 